\title{
Sciendo
}

\author{
BULGARIAN ACADEMY OF SCIENCES
}

CYBERNETICS AND INFORMATION TECHNOLOGIES • Volume 20, No 4

Sofia $\bullet 2020 \quad$ Print ISSN: 1311-9702; Online ISSN: 1314-4081

DOI: $10.2478 /$ cait-2020-0050

\section{Towards a Conceptual Description of Verbs}

\author{
Svetlozara Leseva, Ivelina Stoyanova
}

Department of Computational Linguistics, Institute for Bulgarian Language, Bulgarian Academy of Sciences, 52 Shipchenski prohod Blvd., 1113 Sofia, Bulgaria

E-mails: zarka@dcl.bas.bgiva@dcl.bas.bg

Abstract: Our work is focused on the conceptual description of verbs by employing two main resources - the lexical semantic network WordNet and the conceptual frames from FrameNet. We implement a method for inheritance-based mapping between the two resources by transferring the frame assignments from a hypernym to its hyponyms. We discover that the method performs best for directly related pairs of synsets but deteriorates in assignment at two or more steps. The mapping is then used for enhancing each of the resources by expanding it with new entries and by contributing to the resources' relational structure.

Keywords: FrameNet, WordNet, Frame Semantics, semantic relations.

\section{Introduction and motivation}

Our work aims at mutually enhancing the conceptual description of verbs in WordNet and FrameNet, the two semantic resources used in the study. First, we integrate Frame Semantics as represented in FrameNet with the structured description of verbs in WordNet. We then apply procedures to transfer information from one of the resources into the other using the relations encoded in each of the resources. Below We briefly discuss WordNet and FrameNet with a focus on the semantic relations determining their structure and how these relations are used in the mapping between the resources. Next, we outline the methodology we apply to map WordNet and FrameNet with a recourse to previous alignments and elaborate on how those mappings are expanded by employing the inheritance of conceptual and lexical information. We go on to describe the use of information about the relations that form the structure of the two resources towards enriching their description in several ways: (a) expansion and validation of the mapping; (b) enhancement of FrameNet with information derived from WordNet by suggesting the addition of new lexical units, encoding relations and formulating new conceptual frames; (c) enhancement of WordNet with conceptual information from FrameNet both in terms of expanding it with new entities and improving its relational structure. 


\section{Inter-resource mapping}

\subsection{Resources}

In this work we employ two lexical semantic resources - WordNet and FrameNet. WordNet $[1,2]$ is a large lexical database that represents comprehensively conceptual and lexical knowledge in the form of a network whose nodes denote sets of cognitive synonyms (synsets) interconnected through a number of conceptual-semantic and lexical relations such as synonymy, hypernymy, meronymy, etc. The main relation that determines WordNet's taxonomic structure is the relation of hypernymy (an is-a relation between a more general and a more specific term). FrameNet [3, 4] represents lexical and conceptual knowledge couched in the apparatus of Frame Semantics. Frames are conceptual structures describing particular types of objects, situations, or events along with their components, called frame elements, or FEs [3, 5]. Depending on their semantic obligatoriness and contribution to the conceptual description, FEs may be core, peripheral or extra-thematic [5]: core FEs are the most essential as their configuration makes a frame unique; that is why our focus is on them. Frames are instantiated by Lexical Units (LUs) which are included as part of the description of the relevant frame. In addition, frames are related by means of frame-to-frame relations, which are discussed below. The combination of the two resources is expected to strengthen their individual advantages by bringing together the great lexical coverage and the rich relational structure of WordNet with the detailed conceptual description of the combinatorial potential of lexical units supplied by FrameNet. Our research is directed to the expansion of the mapping of the two resources with the prospect of integrating others, such as VerbNet, PropBank, etc., on the one hand, and to the mutual enhancement of the resources with complementary information, on the other.

\subsection{Compilation of existing mappings}

The understanding that the mapping of semantic resources leads to their mutual enrichment and increases their value in various applications underlies the work of many researchers. Notable efforts in this field include the work of Shi and Mihalcea [6] on the mapping of WordNet, FrameNet and VerbNet, the implementation of FrameNet-to-WordNet mappings, such as WordFrameNet (https://adimen.si.ehu.es/web/WordFrameNet) by Laparra and Rigau [7], MapNet (https://hlt-nlp.fbk.eu/technologies/mapnet) by To ne lli and Pigh in [8], the alignments proposed by Fellb aum and Baker [9] and Ferrande z et al. [10], among others. More enhanced proposals have been made, such as S e mlin k [11] which brings together WordNet, FrameNet and VerbNet with PropBank, and its follow-up Semlink+ that, in addition, includes mapping to Ontonotes [12]. Recently, the interest in linking semantic resources has been rekindled as shown by the efforts of a number of teams. The SynSemClass lexicon (https://ufal.mff.cuni.cz/synsemclass) [13] has marked such an effort towards combining a rich semantic description of verbs with external semantic resources including FrameNet, WordNet, VerbNet, OntoNotes and PropBank. VerbAtlas (http://verbatlas.org/), proposed by Di Fabio, Conia and Navigli [14], is a hand-crafted lexical semantic 
resource which represents synsets as clusters with prototypical argument structures presented as frames, to a large extent inspired by VerbNet roles and semantic restrictions. Le se va, S to y a n ova and Tod orova [15] propose the automatic mapping of WordNet and FrameNet based on inheritance and further refined by the suggestion of procedures for the improvement of the alignment $[19,20]$.

Generally, the inter-resource mappings suffer from limited coverage restricted to aligning corresponding units of the original resources to each other. In the case of FrameNet-to-WordNet mapping, the alignment between corresponding units in the two resources ("direct" mapping) relies on the equivalence or similarity between FrameNet LUs and WordNet synset members (literals), LU definitions and synset glosses, etc. With 155,287 synonyms in 117,659 synsets and more than 246,577 relations, of which 91,631 are instances of the hypernymy relation (https://wordnet.princeton.edu/documentation/wnstats7wn) as compared with 13,640 LUs and 1,875 frame-to-frame relations (https://framenet.icsi.berkeley.edu/fndrupal/current_status) in FrameNet, the discrepancy in the size of the data and the coverage of the mappings between synsets and frames is to be anticipated. The extent of this coverage is reflected to a great degree in previous efforts at mapping WordNet and FrameNet which resulted in 4,306 unique WordNet verb synset-to-FrameNet frame mappings (30.5\% of the total number of verb synsets). Previous efforts on aligning the resources and the initial datasets compiled from the original sources have been described in [15-17].

\subsection{Expanding beyond}

The expansion of the coverage of the inter-resource mapping involves 'digging up' non-explicit semantic information and/or generalising over existing descriptions and transferring them to other units that have not been described yet (e.g., synsets whose literals do not have correspondence as FrameNet LUs) and/or implementing new descriptions.

For instance, VerbAtlas [14] was created bottom-up by clustering synsets according to semantic similarity and defining frames that describe the predicateargument structure and selectional restrictions of each cluster. The predicateargument structures described by the frames are inspired by the VerbNet predicateargument structures but are defined independently (not mapped from VerbNet) based on the WordNet synsets.

Another approach was adopted by B urchardt, Erk and Frank [18], who map FrameNet frames to WordNet synsets. They start from establishing frame-tosynset alignment based on the correspondence between identical synset literals and FrameNet lexical units with the same meaning. Then they go on to propose expansion of the inter-resource coverage by exploring the structural features of the two resources. In particular, they study candidate frames evoked by literals related to a given (target) literal through certain semantic relations (synonymy, hypernymy, antonymy) and assign weights to them.

Another strategy for expansion of the mapping between FrameNet and WordNet proposed by Leseva, Stoyanova and Todorova [15] makes use of the relational structure of the two resources in several ways. The method involves the 
mapping of FrameNet frames to WordNet synsets on the basis of the inheritance of conceptual features in hypernym trees, i.e., by assigning frames from hypernyms to hyponyms; it was further enhanced by implementing and perfecting validation procedures based on the structural properties of the two resources: basically the hypernymy relation in WordNet and several frame-to-frame relations in FrameNet as described in $[19,20]$. We adopt this latter approach.

In addition, we follow earlier work described in [20,21] in identifying the need for new frames to be defined. This is required for those parts of the lexicon that are not yet accounted for in FrameNet. In proposing new frames, we have observed the FrameNet structure, especially in making use of the way in which more concrete frames are described on the basis of more general ones from which they inherit their properties (cf. Section 5.3). We have also made efforts to identify structural inconsistencies and to make up for them, for instance where a causative frame is defined, but not its inchoative counterpart or vice versa (cf. Sections 5.3 and 6). Our purpose is to mutually enrich each of the resources with features and data drawn from the other first by identifying basic units (LUs and literals) that are encoded in one of the resources but missing from the other and finding a way to integrate them, and then by proposing further enhancement, such as addition of relations and formulation of new frames based on observations informed from both resources.

\section{Theoretical aspects of semantic relations within FrameNet and WordNet}

FrameNet and WordNet each have their own relational structure, which is based on semantic relations between language units (in WordNet) or conceptual representations (in FrameNet). The WordNet structure is by far the richer in terms of types and instances of relations; in addition to the conceptual relations it comprises lexical (between literals), derivational and some other types of relations. Although the inventory of relations in the two resources differ in number and scope, at least part of them are grounded in similar universal assumptions, which leads to partial overlap, depending on their definition and the granularity or other specifics of the information encoded in each of the resources. For instance, the Inheritance relation in FrameNet and the hypernymy relation in WordNet both represent a modelling of the is-a relation [5], while the Causativity relation (FrameNet) and the 'causes' relation (WordNet) have equivalent meaning. Here we briefly discuss how the FrameNet frame-to-frame relations translate into WordNet relations, particularly with a view to how this information is used in the mapping and other procedures. A more detailed and in-depth account of the main trends in the correspondence between the relations in the two resources may be found in Le s e va and S t o y a n o va [16].

\subsection{Inheritance}

Inheritance is defined as the strongest relation in FrameNet (Is_Inherited_by and its reverse Inherits_from) which denotes a relationship between a more general (parent) frame and a more specific (child) frame in such a way that the child frame elaborates 
on the parent frame and each semantic fact about the parent must correspond to an equally or more specific fact about the child [5], i.e., generally, there should be a correspondence between entities - frame elements, frame relations and semantic characteristics in the parent and the child frame [22]. Similarly, the main WordNet relation to be considered is hypernymy, which is the principal tree structure (inheritance) organising relation in the resource. We take into account both direct hypernymy (a direct relation between a parent and a child node) and indirect hypernymy (where there are intermediate nodes between the hypernym and the hyponym in question).

Based on this inheritance assumption, we should expect that when there is a pair of WordNet synsets related through the hypernymy relation, then their corresponding frames must be related through a frame-to-frame relation in FrameNet and this relation should be Inheritance. The data only partially confirm this expectation, diverging from it in two ways: (a) there is another frame-to-frame relation which is very strongly favoured for a counterpart of the hypernymy relation, i.e., Using; (b) in some of the cases we find out an inverse relationship, i.e., for a hypernym - hyponym pair, the hyponym is assigned the more general (parent) frame, and the hypernym the child frame in an existing Inheritance relation. This is illustrated by the synset \{crucify:1\}, which is assigned the parent frame Killing, while its hypernym \{execute:1, put to death:1\} is assigned the more specific frame Execution which inherits from Killing. Such cases may stem either from different construals of the senses or from error in the assignment. In this particular case the consideration of the hierarchical relation (hypernymy) between execute and crucify is very informative as to the frame membership; as a result, the human expert has decided that the Execution frame describes crucify's meaning in a more appropriate way.

\subsection{Using}

Another hierarchical relation in FrameNet is Using (Is_Used_by and its reverse Uses). It is defined as a relationship between a child frame and a parent frame in which only some of the FEs of the parent have a corresponding entity in the child, and if such exist, they are more specific [22], hence, it has been suggested that Using may be viewed as a kind of weak Inheritance [22]. The data confirm that indeed the majority of synsets mapped to FrameNet frames with the Using relation are hypernym-hyponym pairs, consider for instance the synset $\{$ travel:1, go:1, move:1, locomote:1\} assigned the frame Motion and its hyponym \{ride:10\} which has been assigned the Operate_vehicle frame that uses the Motion frame. Inverse frame assignment is found with this relation as well; like inverse Inheritance, it represents an interesting theoretical issue with respect to the hierarchical representation of the conceptual properties of verbs.

\subsection{Perspectivisation}

Perspective (couched as the relations Is_Perspectivized and its reverse Perspective_on) indicates that a given situation viewed as neutral may be further specified by means of perspectivised frames that represent (at least two) different possible points-of-view on the neutral situation [5]. As there is a considerable 
correspondence between the frame elements and the conceptual description of the neutral and the perspectivised frames (features shared to a degree with both Inheritance and Using), this relation may translate as the hypernymy-hyponymy relation although it has limited scope in WordNet, with only a couple of frame pairs: for instance, the synset \{attack:1, assail:2\} is assigned the frame Attack which perspectivises Hostile_encounter, the frame assigned to the hypernym \{contend:6, fight:1, struggle:4\}. Apart from the actual WordNet relations, Perspective is found between synsets having a common direct or indirect hypernym with the following pairs of frames: Giving - Transfer, Hostile_encounter - Attack, Transfer-Receiving, Import_export_scenario - Importing, Import_export_scenario - Exporting.

\subsection{Subframe}

Subframe (expressed as Has_Subframe and its reverse Subframe_of) is a relation between a complex frame referring to sequences of states and transitions, each of which can itself be separately described as a frame, and the frames denoting these states or transitions [5]. The definition of Subframe allows for it to be translated as hypernymy in a small number of frames such as Removing and Cause_motion: consider the synset \{expel:1, throw out:1, kick out:1\} which is assigned the frame Removing, itself a subframe of the Cause_motion frame assigned to the hypernym \{move:2, displace:4\}. In more distant structural relations between WordNet synsets with common distant hypernyms, a number of pairs of other frames are found, such as Traversing - Departing, Traversing - Arriving, Intentional_traversing Quitting_a_place, Self_motion - Quitting_a_place, etc. The observations show that although Perspective and Subframe are hierarchical relations, the kind of semantic generalisations underlying them do not correlate well with the WordNet conceptual and lexical relations, inheritance in particular, therefore they have a very limited contribution to the way the mapping is implemented.

\subsection{Precedence}

The Precedence relation (represented by Precedes and its reverse Is_Preceded_by) holds between component subframes of a single complex frame and provides additional information by specifying the chronological ordering of the states and events (subevents) within a complex event [5, 22]. A small number of the Precedence relations are found among antonyms and the majority of the instances is among synsets having a common (direct or indirect) hypernym. The following pairs of frame-to-frame relations are found with antonyms: Placing - Removing, Arriving Departing, Activity_stop - Activity_ongoing. This relation may result in complex structures involving a number of subframes such as the notable example of the Sleep_wake_cycle [23].

The relation does not have a counterpart in the WordNet structure but it may be transferred, thus bringing an additional dimension of semantic description through linking otherwise unrelated subevents and through specifying their temporal ordering. 


\subsection{Inchoativity and Causation}

Inchoativity (represented by Inchoative_of) and Causation (represented by Causative_of) are systematic non-inheritance relationships between stative frames and the inchoative and causative frames that refer to them [5]. Causation has a direct counterpart in the WordNet relation "causes" although these correspondences form a small number of the cases where Causation exists due to the fact that this relation has not been implemented consistently in the two resources. For instance, the lack of the "causes" relation between causative and inchoative senses in WordNet is observed in the hypernym trees whose roots are \{change:1, alter:1, modify:3\} "cause to change; make different; cause a transformation" and \{change: 2 \} "undergo a change; become different in essence; losing one's or its original nature". Using the causative inchoative pairs of frames, e.g., Cause_change and Undergo_change and their inheriting frames - we enrich WordNet with the "causes" relation between respective synsets, and vice versa: define the Causative relation between pairs of frames in FrameNet on the basis of the "causes" relation in WordNet.

\subsection{See also}

See also has no direct semantic meaning but rather serves to differentiate frames which are similar and confusable [5]. It may be construed in quite different ways, which is reflected in the data, through its mapping to a greater variety of WordNet relations: also see, antonymy, verb group, causes, and hypernymy. In the predominant number of cases the synsets whose frames are linked by means of the See_also relation are hypernym-hyponym pairs. For example, the hypernym \{search:4\} "subject to a search" is assigned the frame Scrutiny and the hypernym \{frisk:2 $\}$ "search as for concealed weapons by running the hands rapidly over the clothing and through the pockets" is assigned the frame Seeking. The difference between the two frames is stated as one of different primary focus (to the Sought entity or to the Ground). While this semantic difference is captured by the distinct conceptual structures, it seems to be too fine and does not prevent the construal of \{search:4 $\}$ as the hypernym of $\{$ frisk:2 $\}$. In addition, many of the pairs in a See_also relation are linked through another, more informative relation, e.g., Uses: Bringing Cause_motion, Operate_vehicle - Motion; Inheritance: Self_motion - Motion, Choosing - Deciding; Subframe: Cause_motion - Placing, Cause_motion Removing. In such cases, we consider only the latter.

\section{Inheritance-based mapping}

\subsection{Mapping procedures}

The mapping procedure proposed in [15] and adopted herein uses as a point of departure the initial automatic mapping of 4,306 synsets collected from datasets mapped by other teams and distributed in the community (cf. Section 2). We implemented the assignment proposed by these mappings in iterations, using the relation of inheritance as defined between hypernyms and hyponyms in WordNet, by transferring the hypernyms' frames to their hyponyms in the cases where the 
hyponyms were not directly mapped to FrameNet frames (through the initial mapping). The motivation for adopting such a procedure is that hyponyms elaborate on the meaning of their hypernyms and if a hypernym is assigned a correct frame, this frame should capture the general meaning of the hyponyms as well even if it may provide an underspecified description. By applying the procedure, an extended coverage of 13,226 synsets with an assigned FrameNet frame out of the total of 14,103 verb synsets was obtained as reported in $[15,16]$. We applied the procedure in iterations and at each step a number of frame-to-synset assignments were manually validated in order to ensure the quality of the further applications of the mapping.

Consider Example 1a which illustrates the frame assignment in a WordNet subtree stemming from the synset \{grimace:1; make a face:1; pull a face: 1 \}. The level of indentation shows the level of the hyponyms in the hypernym-hyponym tree. The root of the subtree and eight of its hyponyms were assigned a frame by the initial mapping marked by a " 0 " in the Source column. The root and six of the hyponyms were assigned the correct frame Making_faces and two hyponyms were mapped to other frames, Perception_active and Facial_expression, that are not correct for the particular senses of the synsets; for instance Facial_expression is assigned only to nouns denoting such expressions. Five of the direct and indirect hyponyms of the root were not mapped by the initial mapping most likely because the respective literals are not included as lexical units in FrameNet (Example 1b below shows all the LUs in this FrameNet frame). The frame Making_faces was assigned on the basis of inheritance from the hypernym. The number in the Source column indicates the number of steps on which the assignment is made; " 1 " means that the hypernym's frame is assigned directly from the parent synset. The last column shows the result of the manual validation and the corrections made by an expert. In this particular case, out of 14 synsets, the initial mapping succeeded to make seven correct and two wrong assignments and could not assign a frame to five synsets; the frame was correctly predicted for the latter by the inheritance-based assignment.

Example 1a. WordNet subtree of \{grimace:1; make a face:1; pull a face:1\} Synset

- - grimace:1; make a face: 1 ; pull a face: 1 ;

- - - pout:2; mop:2; mow:2;

- - squint:1; squinch:3;

- - - frown:1; glower:2; lour:3; lower:5;

-- scowl: 1 ;

- - smile: 1 ;

- - - dimple:2;

- - - smirk:1; simper:1;

----- fleer: 1 ;

--- grin: 1

---- sneer: 2 ;

- - - beam: 1 ;

- - - wince: 2 ;

- - - screw up:4; Frame assigned Source Expert

Making_faces $0 \quad$ OK

Making faces $0 \quad \mathrm{OK}$

Perception active 0 Making_faces

Making_faces $0 \quad \mathrm{OK}$

Making_faces $0 \quad$ OK

Making_faces $0 \quad$ OK

Making_faces 1 OK

Making_faces $0 \quad$ OK

Making_faces 1 OK

Making_faces $0 \quad$ OK

Facial expression 0 Making_faces

Making_faces 1 OK

Making_faces 1 OK

Making_faces 1 OK 
Example 1b. LUs that evoke the Making_faces frame in FrameNet

$\begin{array}{lll}\text { frown.v } & \text { Created Lexical entry } & \text { Annotation } \\ \text { grimace.v } & \text { Created Lexical entry } & \text { Annotation } \\ \text { grin.v } & \text { Created Lexical entry } & \text { Annotation } \\ \text { pout.v } & \text { Created Lexical entry } & \text { Annotation } \\ \text { scowl.v } & \text { Created Lexical entry } & \text { Annotation } \\ \text { smile.v } & \text { Created Lexical entry } & \text { Annotation } \\ \text { smirk.v } & \text { FrameNet1_Sent Lexical entry } & \text { Annotation }\end{array}$

In addition, we also adopted a set of automatic procedures aimed at evaluating the consistency of the mapping against a set of criteria as proposed by $[15,19,20]$. These include discrepancies between the semantic relations connecting synsets in WordNet and the semantic relations between their corresponding assigned frames; the procedures suggest potentially wrong assignments or problems in the structure of either resource and are paid additional attention.

Finally, we adopted a set of procedures aiming at suggesting more specific or appropriate frames than the ones inherited from the hypernyms by employing the frame-to-frame relations in FrameNet and synset-to-synset relations in WordNet as proposed by [19], which were further extended and perfected in [20]. The motivation for the implementation of these procedures was the observation that at $2+$ steps the precision of the inheritance mapping tends to deteriorate (see Section 4.2). Two of the identified reasons for this are (i) that the sense of the hyponym becomes too remote from that of the hypernym and its appropriate description requires a more specific frame, or (ii) that certain semantic information gets profiled in the hyponyms and needs to be captured by a different frame.

The process of validation led also to identifying missing nodes (frames) and relations in the internal frame structure of FrameNet and inconsistencies in WordNet, especially in the domain of causativity/inchoativity. Finally, with the advancement and validation of more frame-to-synset assignments, we have become aware of the need to define new frames for parts of the lexicon not yet accounted for in FrameNet. Part of these findings are tackled below.

\subsection{Evaluation of the inheritance-based mapping}

We evaluate the precision of the inheritance-based mapping on the set of validated frame-to-synset assignments (5,025 verb synsets) with a view to the possibilities for enriching both resources with information from the other at various levels. Along with the relation of equivalence, where a hypernym and a hyponym are assigned the same frame (SAME) and the relation of inheritance where a hyponym is assigned a frame that is in one of the hierarchical relations (Inheritance, Using, Perspective, Subframe, cf. Section 3) with the frame assigned to the parent synset (INHERITANCE), the data show other types of relationship as well. One is INVERSE INHERITANCE where the hyponym is assigned a more general frame and the hypernym - a more specific one.

In the case of an indirect relation (INDIRECT), a hypernym and a hyponym are assigned a pair of frames where the two are connected indirectly through an intermediate frame: e.g., \{borrow:1\} "get temporarily" is assigned the frame 
Borrowing and its hypernym \{get:1, acquire:1\} "come into the possession of something concrete or abstract" is assigned the frame Getting. Borrowing inherits from Getting through the Receiving frame, so that the three frames are involved in the following inheritance relation (from more general to more specific): Getting > Receiving > Borrowing. In WordNet a relevant synset \{receive:1, have:15\} "get something; come into possession of", which is assigned the frame Receiving, is a cohyponym of $\{$ borrow: 1$\}$, i.e., a hyponym of $\{$ get, acquire: 1$\}$. Such cases, if linguistically motivated, may indicate a different way of constructing the lexical hierarchy; they are nonetheless inspected manually in order to validate that this is indeed the case.

In a number of instances the frames assigned to a hypernym and its hyponyms are in a different (non-hierarchical) relation (OTHER DIRECT). Most of them were revised and found to be the result of wrong assignment in the initial mapping, especially one between a hypernym assigned a causative or an inchoative frame and a hyponym assigned a frame in the other domain.

An interesting case is the lack of relationship (NO RELATION) between the frames assigned to a hypernym and a hyponym. Consider the following hypernymhyponym chain: \{work:2; do work:1\} "be employed" > \{serve:2\} "do duty or hold offices; serve in a specific function" $>\{$ staff: 2$\}$ "serve on the staff of" and the frames assigned to the synsets from hypernym to hyponym Being_employed > Serve_in_capacity > Working_a_post. The three frames, however, are not related to each other through any relation, even indirectly, although all of them are related to employment and may be argued to belong to the same situational scenario. We leave it for future work to elaborate on the possibilities of encoding frame-to-frame relations in such cases.

We implement a scoring system for evaluating the precision of the assignments, which we measure as the distance between the automatically assigned frame using the inheritance-based mapping and the manually validated frame. The score is calculated in the following way: (a) 1.0 for each exact match; (b) 0.750 for each pair that is related through a direct inheritance relation; (c) 0.625 for each pair that is related through a direct inheritance relation in two steps; (d) 0.500 for a pair that is related through a direct non-hierarchical relation; (e) 0.375 for a pair that is related through a non-hierarchical relation in two steps (the second step might involve inheritance); (f) 0.250 for a pair of frames in inverse inheritance relation; (g) 0.125 for a pair of frames related to each other through a third frame (indirect relation); (h) 0.000 for non-related pairs of frames.

The evaluation is performed on three datasets corresponding to the transferring of frames from hypernyms to hyponyms in one (directly), two (to the hyponyms of the hyponyms) and three steps. The results in Table 1 are consistent with the observations that in two or more steps the quality of the assignment deteriorates. These results support our choice of the iterative approach where manual validation is performed at each step after the automatic assignment of frames to synsets. In this way, we control the quality of the resulting dataset by reducing the depth of automatic inheritance-based mappings. 
Table 1. Scoring system for the evaluation of the precision of WordNet-to-FrameNet inheritance-based mapping

\begin{tabular}{|l|r|r|r|r|}
\hline Scoring system & Score & One-step & Two-step & Three-step \\
\hline Same & 1.000 & 1.091 & 340 & 85 \\
\hline Inheritance & 0.750 & 33 & 67 & 36 \\
\hline Inheritance (in two steps) & 0.625 & 23 & 45 & 62 \\
\hline Other direct & 0.500 & 145 & 208 & 97 \\
\hline Other direct (in two steps) & 0.325 & 150 & 301 & 240 \\
\hline Inverse_inheritance & 0.250 & 89 & 74 & 43 \\
\hline Indirect & 0.125 & 0 & 0 & 0 \\
\hline No found relation & 0.000 & 181 & 291 & 206 \\
\hline Total number of assignments & & $\mathbf{1 . 7 1 2}$ & $\mathbf{1 . 3 2 6}$ & $\mathbf{7 6 9}$ \\
\hline Average precision & & $\mathbf{0 . 7 4 4}$ & $\mathbf{0 . 4 8 2}$ & $\mathbf{0 . 3 7 5}$ \\
\hline
\end{tabular}

\section{Towards expanding the semantic description within FrameNet}

The linking between FrameNet and WordNet provides the possibility for enhancing FrameNet at several levels: (i) expanding the LU inventory by suggesting literals in successfully mapped synsets that are not included in FrameNet in the given sense to be added to the respective frame(s); (ii) introducing LU-to-LU relations that correspond to literal-to-literal or synset-to-synset relations in WordNet; (iii) proposing frame-to-frame relations on the basis of synset-to-synset relations; (iv) defining new frames for different groupings of verbs.

\subsection{Expanding and structuring the LU inventory}

The mapping of the synset \{grimace:1; make a face:1; pull a face:1\} and its hyponyms to the Making_faces frame (Example 1a) enables us to enhance the FrameNet frame by: (i) expanding its lexical coverage by 16 LUs and the lexical and relational information that pertains to them (doubling the number of LUs; the literals in bold in Example 1a are the ones found as LUs in the respective sense in FrameNet) and (ii) introducing hierarchical and other relations among LUs by incorporating the hypernymy, synonymy and other relation from WordNet, thus bringing another dimension to the internal organisation of the FrameNet frames, where possible.

Originally, there were 5,235 verbal LUs (out of a total of 13,701 LUs) assigned to frames within FrameNet. As a result of the mapping of frames to synsets, we were able to contribute another 6,357 verbal LUs to a total of 452 frames (from the subset of the manually validated assignments). The candidate LUs within each frame were transferred along with the WordNet relations (where available) with both candidate and already existing LUs, e.g., in the Making_faces frame the candidate LU simper is added with the synonymy relation to the existing LU smirk and with the hyponymy relation to smile. Table 2 shows the new LU-to-LU relations transferred from WordNet. 
Table 2. Distribution of synset relations between the LUs in total across all frames

\begin{tabular}{|l|c|}
\hline TYPE OF RELATION & Number of related pairs \\
\hline SYNONYMS & 4,570 \\
\hline SISTERS (Common hypernym) & 31,859 \\
\hline HYPERNYM - HYPONYM & 2,970 \\
\hline VERB_GROUP & 135 \\
\hline ANTONYM & 118 \\
\hline ALSO_SEE & 57 \\
\hline
\end{tabular}

Sisterhood is the predominant relation, which means that the LUs in this relation have a direct common hypernym. This is to be expected and confirms the observation that synsets belonging to the same subtree are frequently assigned the same frame. The most informative relations are synonymy (a pair of LUs belong to the same synset), hypernymy (one LU belongs to the hypernym synset with respect to the other) and antonymy (two LUs belong to antonymous synsets). Some of the frames with the largest number of LUs are: Statement (447 newly added LUs), Stimulate_emotion (445 new LUs), Self_motion (383 new LUs), etc.

\subsection{Definition of new relations}

Another aspect of the linking of the two resources is increasing the internal connectivity of the FrameNet structure by introducing more instances of the existing frame-to-frame relations or defining new ones on the basis of the relations available in WordNet.

So far the most reliable way to enrich FrameNet automatically with new relations is to identify causative-inchoative pairs of frames based on the existing "causes" relation between the pair of synsets frames have been assigned to. For example, we identify pairs of frames such as the causative Cause_to_wake assigned to the synset \{resuscitate:2; revive:3\} and the inchoative Waking_up assigned to \{come to:1; revive:1; resuscitate:1\} where the synsets exhibit the "causes" relation in WordNet but the FrameNet frames do not. This observation leads to the suggestion for restructuring the relevant frame-to-frame relations in FrameNet: Cause_to_wake is causative of the stative frame Being_awake which in turn Is_Preceded_by Waking_up, whereas it should be in a causative relation with the inchoative frame (Waking_up) and there should be an inchoative relation between the inchoative frame and the stative frame, i.e., Cause_to_wake Is_causative_of Waking_up Is_inchoative_of Being_awake.

Antonymy between synsets can also yield possible relations between the frames assigned to them. For example, we observe antonymy between \{continue:5; uphold:3; carry on:4; bear on:4; preserve:4\} assigned the frame Cause_to_continue and \{discontinue:2; stop:13; cease:2; give up:12; quit:5; lay off:2\} mapped to Activity_stop. When defining the relations between these frames, we need to take into consideration causation as well since one of the frames is causative (Cause_to_continue). A similar example involves the frames Activity_start and Cause_to_end. 
Antonyms often express two different actions within the same situation. In some cases these complex situations are formulated as a complex frame, i.e., Import_export_scenario, Cycle_of_existence_scenario, Crime_scenario, etc. These offer an additional layer of generalisation which facilitates grouping related frames based on the type of situation they describe, and will contribute to the relational structure within FrameNet.

\subsection{Definition of new frames}

There are a couple of directions for expanding the coverage by introducing new frames. The first one involves the formulation of frames that elaborate on more general ones in a way that is consistent with the definition of already existing frames. Such elaborations are especially relevant for frames that have a very general meaning that is specialised in their inheriting frames. This is the case with Cause_change and its inchoative counterpart Undergo_change. As Le s e va et al. [21] make the case, frames elaborating on changes in various attributes, such as temperature (Cause_temperature_change), consistency (Cause_change_of_consistency), phase (Cause_change_of_phase), strength (Cause_change_of_strength), among others, have been defined in FrameNet, while other, equally specific properties, such as colour, taste, chemical composition, etc., have not been accounted for yet. Such new frames need to be modelled on already formulated ones, e.g., the definition of Cause_chemical_reaction, Cause_change_colour, Cause_change_taste should follow those of Cause_change_of_consistency and Cause_change_of_phase with which they most closely correspond.

A second direction for enriching the structure of FrameNet is defining frames that are predictable from the FrameNet structure but have not been implemented. A notable example is the lack of frame correspondences between causative and inchoative parts of the lexicon where either of the members may be missing. We take as a model pairs of frames, such as Cause_change and Undergo_change, among many others, where the causative frame is related to the inchoative frame by means of the Causative_of relation; we then proceed to define a new causative or inchoative frame where one must exist and link it to its counterpart by means of this relation. For instance, the frame Cause_change_of_strength assigned to \{strengthen:1, beef up:1, fortify: 1$\}$ "make strong or stronger" does not have an inchoative counterpart that should be assigned to \{strengthen:2\} "gain strength". In other cases, the causative frame may be missing: Change_direction, Motion_directional, Self_motion do not have causative correspondences although this distinction is made for their parent frame Motion (with its counterpart Cause_motion). Thus, for instance, $\{$ march:3 $\}$ "walk fast, with regular or measured steps; walk with a stride" is assigned the frame Self_motion, but there is no corresponding frame to account for $\{$ march: 2$\}$ "force to march" and other verbs describing self propelled motion brought about through the action of another participant.

In yet other cases, the causative frames embrace the meaning of the noncausative one. For instance, the Filling frame describes the synset \{fill:1, fill up:1, make full:1 1 "make full, also in a metaphorical sense" and a large class of verbs of filling, but there is no frame that corresponds to the inchoative meaning 
\{fill:2, fill up:2\} "become full" and its hyponyms. The same holds for Hiding_objects, which describes the synsets stemming from \{hide:1, conceal:1\} "prevent from being seen or discovered" but does not have a counterpart that accounts for the subtree stemming from \{hide:2, hide out: 1 \} "be or go into hiding; keep out of sight, as for protection and safety". In such cases, we also suggest the definition of new frames modelled on the respective existing frame, following the general structure.

So far 29 new frames have been defined covering a total of 439 synsets.

\section{Expanding the semantic description in WordNet}

The work on expanding the semantic description of WordNet by employing the conceptual information from FrameNet relies on the following assumption: synsets that have been assigned the same frame or a pair of related frames are expected to exhibit a semantic relation as well. Generally, this translates into the condition that a semantic relation in one of the resources should normally be reflected into a corresponding semantic relation in the other. Since this is true for verb synsets within the same WordNet tree, the hypothesis should be checked for synsets in different WordNet trees which might exhibit an undefined semantic relation.

\subsection{New lexical entities}

We can employ the information from FrameNet to expand the lexical base of WordNet in two directions: (a) by adding new literals to existing synsets; (b) by defining new synsets to the end of expanding the lexical data and improving the relational structure in terms of coverage and consistency.

In a similar way as enriching frame description by adding new LUs to frames using literals from synsets sharing that frame, we apply the opposite procedure and consider candidate LUs that can be added to particular synset based on the similarity between the synset's gloss and the LU's definition.

Using automatic consistency checking procedures we were able to identify possible lexical or conceptual "gaps" in the WordNet structure. These occur in cases where some synsets combine synonyms that exhibit different semantics and thus require different frames. For example, the synset \{blacken:1\} defined as "make or become black" collapses a causative \{blacken:1a\} "make black", and an inchoative sense: \{blacken:1b\} "become black". These in fact may be expressed in other languages by different lexical units; e.g., in Bulgarian and other Slavic languages the non-causative is often morphologically related to the causative (formed by the same root) but is marked with the particle se.

\subsection{New relations}

We consider the relations between frames in order to define and implement new synset-to-synset relations in WordNet. Firstly, the assignment of frames to synsets divides synsets into classes based on their conceptual structure and the number and types of the involved frame elements applicable to verb literals within synsets. The 
classes of verbs formed by the frame assignments (e.g., verbs with the frame Cause_change_of_consistency, Cause_change_of_shape, etc.) can be generalised based on frame-to-frame inheritance relations (e.g., lump together both classes to the more general frame Cause_change) or further refined, depending on the specific research purposes. In some cases groups of verbs correspond roughly to hypernymhyponym (sub)trees in the WordNet structure.

Table 3 shows synsets that are assigned related frames but the synsets themselves are in separate WordNet (sub)trees and have a distant or no semantic relations among them. As the data shows, these pairs comprise the largest proportion of the data. They are potential candidates for new WordNet relations. In particular, since we are interested in the more specific frame-to-frame relations which provide knowledge beyond inheritance that may be transferred onto new WordNet relations, we consider relations such as Causation and Inchoativity, Subframe, Perspective, and Precedence. We look both at directly related frames and at cases of an indirect relation where the frames are both Perspectives, Subframes, etc. of a third frame.

Table 3. Distribution of synset pair relations for particular frame relations existing between the pair of frames assigned to the synsets

\begin{tabular}{|l|c|c|c|c|c|}
\hline Relation & CAUSATION & INCHOATIVITY & PERSPECTIVE & SUBFRAME & PRECEDENCE \\
\hline verb_group & 19 & - & - & - & - \\
\hline antonym & 1 & - & - & - & 3 \\
\hline also_see & 4 & - & - & 2 & 2 \\
\hline causes & 30 & - & - & - & - \\
\hline hypernym & 22 & - & 2 & 2 & - \\
\hline sisters & 66 & - & - & 53 & 4 \\
\hline INDIRECT & 336 & - & - & 7 & 22 \\
\hline $\begin{array}{l}\text { NO } \\
\text { RELATION }\end{array}$ & 12,175 & 161 & 31 & 6,068 & 1,063 \\
\hline
\end{tabular}

The correspondence between "antonym", "hypernym", "sisters" and the causative relation (column 2) are due to errors that were manually corrected. The examples with "verb_group" and "also_see" represent semantic similarity and in most cases stands for an unspecified causative relation, which is then added.

The possible candidate pairs for a given relation between more distant synsets, i.e., related through at least one intermediate node or unrelated (INDIRECT and NO RELATION) are a large number, and require further automatic procedures to filter them down to plausible candidates and facilitate manual validation. To this end, various morphological and semantic procedures can be used. With regard to causation, we reduce possible candidates by selecting only the ones that either: (a) share a morphologically identical verb in both synsets in English; (b) have a pair of a causative verb and a same-root verb with the particle se in Bulgarian. Consider, for instance, the synset \{decrease:3; lessen:3; minify:1\} "make smaller", which has been assigned the frame Cause_change_of_position_on_a_scale. Initially, 17 possible candidates were identified which were assigned the non-causative frame Change_position_on_a_scale. By applying the procedures, the candidates are 
reduced to the synset \{decrease:1; diminish:1; lessen:1; fall:4\} "decrease in size, extent, or range" identified on the basis of the literal-to-literal correspondence between lessen:3 and lessen:1 in English, as well as between ponizhavam:1 and ponizhavam se:2 in Bulgarian. In this way, we reduce the possible candidates 12 times (from 12,175 to 989). Similar procedures can be designed for other relations by considering the semantic and morphological properties of verbs, such as prefixation, suffixation, etc., possibly combining information from more than one language.

\section{Conclusions and future work}

The work envisaged in the near future is aimed at providing further validation of the frame assignment to verb synsets in WordNet. A challenging prospective research will be to devise new frames that provide description of parts of the verb lexicon that have not yet been tackled in FrameNet as well as of parts of the Bulgarian verb lexicon that have no English counterparts.

A further goal is to employ the obtained linked resource in tasks such as semantic role labelling, event detection, syntactic parsing, and machine translation, among others. The mapping between WordNet and FrameNet as well as the newly devised frames will be made available to the research community.

Acknowledgements: This study has been carried out as part of the Project "Towards a Semantic Network Enriched with a Variety of Semantic Relations" funded by the National Science Fund of the Republic of Bulgaria under the Fundamental Scientific Research Programme (Grant Agreement 10/3 of 14.12.2016).

\section{References}

1. Mille r, G. WordNet: A Lexical Database for English. - Communications of the ACM, Vol. 38, No 11, pp. 39-41.

2. C. Fellbaum, Ed. WordNet: An Electronic Lexical Database. Cambridge, MA, MIT Press.

3. B a k e r, C. F., C. J. F 111 m or e, J. B. L o w e. The Berkeley FrameNet Project. - In: Proc. of 36th Annual Meeting of the Association for Computational Linguistics and 17th International Conference on Computational Linguistics (COLING-ACL'98), 10-14 August 1998, Montreal, Canada, pp. 86-90.

4. B a k e r, C. FrameNet, Present and Future. - In: Proc. of 1st International Conference on Global Interoperability for Language Resources (ICGL-2008), Hong Kong, January 2008, pp. 12-17.

5. Ruppenhofer, J., M. Ells w orth, M. R. L. Petruck, C. R. Johnson, C. F. B aker, J. S c h e f f c z y k. FrameNet II: Extended Theory and Practice (Revised November 1, 2016). https://framenet2.icsi.berkeley.edu/docs/r1.7/book.pdf

6. Shi, L., R. Mi h l c e a. Putting Pieces Together: Combining FrameNet, VerbNet and WordNet for Robust Semantic Parsing. - In: A. Gelbukh, Ed. Computational Linguistics and Intelligent Text Processing (CICLing'05). Lecture Notes in Computer Science. Vol. 3406. Berlin, Heidelberg, Springer, 2005, pp. 100-111.

7. L a p a r r a, E., G. R i g a u. eXtended WordFrameNet. - In: Proc. of 7th International Conference on Language Resources and Evaluation (LREC'10), 17-23 May 2010, Valletta, Malta, pp. 1214-1219.

8. To n elli, S., D. P i g h i n. New Features for Framenet - Wordnet Mapping. - In: Proc. of 13th Conference on Computational Natural Language Learning (CoNLL’09), Boulder, USA, 2009. 
9. Fe 11 b a u m, C., C. F. B a ker. Can WordNet and FrameNet be Made "Interoperable"? - In: Jonathan Webster, Nancy Ide, Alex Chengyu Fang, Eds. Proc. of the 1st International Conference on Global Interoperability for Language Resources, Hong Kong, City University, pp. 67-74.

10. Fe rr a n d e z, O., M. E 11 s w or t h, R. M u n o z, C. F. B a k e r. Aligning FrameNet and WordNet Based on Semantic Neighborhoods. - In: Proc. of 7th Conference on Language Resources and Evaluation (LREC'10), 17-23 May 2010, Valletta, Malta, pp. 310-314.

11. P a $1 \mathrm{~m}$ e r, M. Semlink: Linking PropBank, VerbNet and FrameNet. - In: Proc. of Generative Lexicon Conference, pp. 9-15.

12. Palmer, M., C. B on ial, D. Mc Carthy. SemLink+: FrameNet, VerbNet and Event Ontologies. - In: Proc. of Frame Semantics in NLP: A Workshop in Honor of Chuck Fillmore (1929-2014), Baltimore, USA, ACL, pp. 13-17.

13. U r e š ová, Z., E. Fu cík ová, E. H a j i c ová, J. H a j i c. SynSemClass Linked Lexicon: Mapping Synonymy between Languages. - In: Proc. of Globalex Workshop on Linked Lexicography, Language Resources and Evaluation Conference (LREC'20), Marseille, 11-16 May 2020, pp. 10-19.

14. Di Fabio, A., S. Con i a, R. N a vigli. VerbAtlas: A Novel Large-Scale Verbal Semantic Resource and its Application to Semantic Role Labeling. - In: Proc. of 2019 Conference on Empirical Methods in Natural Language Processing and the 9th International Joint Conference on Natural Language Processing, Hong Kong, China, 3-7 November 2019, Association for Computational Linguistics, pp. 627-637.

15. Le s e va, S., I. S to y a n ova, M. Tod or o va. Classifying Verbs in WordNet by Harnessing Semantic Resources. - In: Proc. of 3rd International Conference Computational Linguistics in Bulgaria (CLIB'18), Institute for Bulgarian Language, 2018, pp. 115-125. ISSN:2367-5675.

16. L e s e va, S., I. S t o y a n o v a. Enhancing Conceptual Description through Resource Linking and Exploration of Semantic Relations. - In: Proc. of 10th Global Wordnet Conference, 23-27 July 2019, Wrocław, Poland. Oficyna Wydawnicza Politechniki Wrocławskiej, 2019, pp. 280-289.

17. L e s e v a, S., I. S t o y a n o v a. Beyond Lexical and Semantic Resources: Linking WordNet with FrameNet and Enhancing Synsets with Conceptual Frames. - In: S. Koeva, Ed. Towards a Semantic Network Enriched with a Variety of Semantic Relations. Prof. Marin Drinov Academic Publishing House of Bulgarian Academy of Sciences, 2020.

18. B u r c h a rd t, A., K. Er k, A. Fran k. A WordNet Detour to FrameNet. - In: Sprachtechnologie, mobile Kommunikation und linguistische Resourcen. Computer Studies in Language and Speech, Vol. 8, Peter Lang, Frankfurt, Germany, 2005.

19. S t o y a n o va, I., S. Le s e va. A Structural Approach to Enhancing WordNet with Conceptual Frame Semantics. - In: Proc. of Recent Advances in Natural Language Processing (RANLP'19), Varna, Bulgaria, 2-4 September 2019, pp. 629-637.

20. Leseva, S., I. S to y a nova. Consistency Evaluation towards Enhancing the Conceptual Representation of Verbs in WordNet. - In: Proc. of 4th International Conference "Computational Linguistics in Bulgaria" (CLIB'20), Institute for Bulgarian Language Bulgarian Academy of Sciences, 2020, 165-175. ISSN: 2367-5675 (online).

21. Le s e va, S., I. S t o y a n o va, M. Tod or o va, H. K u k ov a. Frame Specialisation Motivated by Inter-Frame Relations in FrameNet. - In: Proc. of 14th International Conference on Linguistic Resources and Tools for Natural Language Processing, Cluj-Napoca, 18-20 November 2019, Editura Universității „Alexandru Ioan Cuza” din Iași, 2019, pp. 167-178. ISSN:1843-911X.

22. P e t r u c k, M. R. The Components of FrameNet. Tutorial at NAACL 2015. International Computer Science Institute, Berkeley, CA. [01.06.2020]. http://naacl.org/naacl-hlt-2015/tutorial-framenet-data/FNComponentsMRLP.pdf

23. Petru ck, M. R., G. de Melo. Precedes: A Semantic Relation in FrameNet. - In: Proc. of Workshop on Language Resources for Public Security Applications, 2012, pp. 45-49.

Received: 02.11.2020; Accepted: 09.11.2020 (fast track) 